\section{P55 INCIDENCE OF ISCHAEMIC HEART DISEASE IN PEOPLE WITH AND WITHOUT HISTORY OF SCHIZOPHRENIA AND BIPOLAR DISORDER IN SCOTLAND 2001-2015}

${ }^{1} \mathrm{CA}$ Jackson*, ${ }^{2} \mathrm{~J}$ Kerssens, ${ }^{3} \mathrm{D}$ Smith, ${ }^{2} \mathrm{~K}$ Licence, ${ }^{3} \mathrm{~S}$ Mercer, ${ }^{1} \mathrm{SH}$ Wild. ${ }^{1}$ Institute of Population Health Sciences and Informatics, University of Edinburgh, Edinburgh, UK: ${ }^{2}$ Information Services Division, NHS National Services Scotland, Edinburgh, UK; ${ }^{3}$ Institute of Health and Wellbeing, University of Glasgow, Glasgow, UK

10.1136/jech-2018-SSMabstracts. 179

Background Major mental illness has previously been identified as a risk factor for ischaemic heart disease (IHD) and stroke. It is not clear whether these associations differ by age, sex, socio-economic status or time period.

Methods Dynamic cohorts of people with no history of hospital admission for IHD or stroke and either a record of hospital admission for schizophrenia or bipolar disorder or no history of hospital admission for severe mental illness were created using Scottish population-based national records. Absolute incidence rates were calculated for all three cohorts and Poisson regression models were used to estimate relative risks (RR) for IHD and stroke events identified using hospital admission and mortality records), stratified by or adjusted for age, sex, time period and area-based deprivation index.

Results Between 1991 and 2015, the absolute risk of IHD and stroke decreased in people with schizophrenia and bipolar disorder and in those without mental illness, but there was little change in the relative risk, comparing those with and without mental illness. IHD risk was about two-fold and $60 \%$ higher in people with schizophrenia and bipolar disorder, respectively, compared to those without mental illness (age, sex and deprivation-adjusted RR for schizophrenia in 1991: RR 1.90, 95\% CI 1.38, 2.58; and in 2015: RR 2.11, 1.63, 2.74); adjusted RR for bipolar disorder in 1991: RR 1.58, $95 \%$ CI 1.48, 1.69; and in 2015: RR 1.64, 1.52, 1.78). Similar results were observed for stroke incidence. RRs were similar across age groups. However, there was an interaction with sex (higher RRs for women than men) and with deprivation (higher RRs in more deprived groups in people with schizophrenia and bipolar disorder than in those without mental illness).

Conclusion History of hospital admissions with schizophrenia or bipolar disorder is associated with higher incidence of IHD and stroke, with stronger associations: in women than men; and in more than less deprived populations, but no evidence of interaction with age and no change in RRs over time.

Better prevention of IHD and stroke is needed in people with major mental illness. Strengths lie in the use of national routine datasets, which provide sufficient power for the study of relatively rare mental health disorders and allow analysis of time trends. The work is however limited use of hospital admission data to define mental illness and the absence of risk factor data.

\section{P56 MENTAL HEALTH OUTCOMES IN OFFSPRING EXPOSED TO ANTENATAL CORTICOSTEROIDS FOR THREATENED PRETERM LABOR: A SYSTEMATIC REVIEW OF THE LITERATURE}

EBC Ndege, A Ayorinde*, S Bhattacharya. Master of Public Health, School of Medicine, Medical Sciences and Nutrition, University of Aberdeen, Aberdeen, UK
Background Antenatal corticosteroids (AC), recommended by the National Institutes of Health Consensus Development panel, are widely administered in pregnant women with impending preterm irth to hasten maturity of the lungs in the fetus. In preterm infants, treatment with $\mathrm{AC}$ has been shown to reduce neonatal death and morbidity, however, less is known about the longer term mental health outcomes in the exposed offspring. Our aim was to assess the effects of AC on the mental health of the exposed offspring as compared to the unexposed offspring.

Methods Studies were retrieved from electronic searches of Medline, Embase, Cochrane Central Register of Controlled Trials, PsychINFO and Pubmed with no language or date restrictions. All databases were searched from inception to May 2017. The search strategy was developed in Ovid Medline and adapted as necessary for other databases to identify the relevant studies by utilizing a combination of Medical Subject Headings terms for antenatal, corticosteroids, offspring and adverse mental outcomes. Reference lists and the bibliographies of the retrieved articles were searched by hand. We included comparative studies that assessed any mental health outcomes in offspring with threatened preterm birth whose mothers were exposed to AC. Relevant studies were selected using pre-defined inclusion and exclusion criteria. Study characteristics were extracted using a pre-defined and piloted form and quality assessed using the Critical Appraisal Skills Programme criteria. A narrative synthesis of the data was conducted to compare mental health outcomes between offspring exposed to AC and unexposed to AC.

Results A total of 2329 unique citations were identified, 28 full-text publications were screened and 11 cohort studies met the inclusion criteria for this review. The 11 studies included a total number of 18261 participants in which 13131 were exposed to AC. All the studies compared offspring exposed to AC to those who were unexposed with variation in the courses of AC exposure in terms of single or repeat courses. Nine studies assessed neurodevelopmental impairment in offspring aged $<3$ years. Two studies reported adverse effects of AC in offspring aged $\geq 3$ years; behavioral problems in 3-yearolds and depression, anxiety, avoidant personality problems in 22-39 year-olds.

Conclusion The results of this review demonstrate insufficient evidence to conclude that AC exposure in offspring with threatened preterm birth is associated with adverse mental health outcomes. There is a need for conducting further research studies with long-term follow up of exposed offspring to ascertain the association.

\section{\begin{tabular}{l|l} 
P57 & ABSTRACT WITHDRAWN
\end{tabular}}

